\title{
On J-Cone Metric Spaces over a Banach Algebra and Some Fixed-Point Theorems
}

\author{
Jerolina Fernandez, ${ }^{1}$ Neeraj Malviya, ${ }^{2}$ Vahid Parvaneh $\left(\mathbb{D},{ }^{3}\right.$ Hassen Aydi $\triangle\left(\mathbb{D},{ }^{4,5}\right.$ \\ and Babak Mohammadi ${ }^{6}$ \\ ${ }^{1}$ Department of Science, The Bhopal School of Social Sciences, 462024, Bhopal, M.P., India \\ ${ }^{2}$ Department of Mathematics, Government College, Timarni, M.P., India \\ ${ }^{3}$ Department of Mathematics, Gilan-E-Gharb Branch, Islamic Azad University, Gilan-E-Gharb, Iran \\ ${ }^{4}$ Université de Sousse, Supérieur d'Informatique et des Techniques de Communication, H. Sousse 4000, Tunisia \\ ${ }^{5}$ China Medical University Hospital, China Medical University, Taichung 40402, Taiwan \\ ${ }^{6}$ Department of Mathematics, Marand Branch, Islamic Azad University, Marand, Iran
}

Correspondence should be addressed to Vahid Parvaneh; zam.dalahoo@gmail.com and Hassen Aydi; hassen.aydi@isima.rnu.tn

Received 22 October 2020; Revised 9 January 2021; Accepted 13 January 2021; Published 1 February 2021

Academic Editor: Remi Léandre

Copyright (c) 2021 Jerolina Fernandez et al. This is an open access article distributed under the Creative Commons Attribution License, which permits unrestricted use, distribution, and reproduction in any medium, provided the original work is properly cited.

In the present paper, we define $J$-cone metric spaces over a Banach algebra which is a generalization of $G_{p_{b}}$-metric space $\left(G_{p_{b}}\right.$-MS) and cone metric space (CMS) over a Banach algebra. We give new fixed-point theorems assuring generalized contractive and expansive maps without continuity. Examples and an application are given at the end to support the usability of our results.

\section{Introduction}

The notion of a generalized partial $b$-metric (for short, a $G_{p_{b}}$ -metric) space was introduced by Hussain et al. [1] in 2014 by generalizing the notions of a $G$-metric space (G-MS) and a partial $b$-metric space. They studied the related topological properties and provided fixed point theorems for some contractive maps.

Huang and Zhang [2] generalized the notion of metric spaces to a CMS. Later, the interesting concept of a cone metric space over a Banach algebra (for short, CMS over a $\mathrm{BA}$ ) was proposed by Liu and $\mathrm{Xu}$ [3], by replacing a CMS with a CMS over a BA. Motivated by these ideas, many authors further considered a CMS over a BA (see $[4,5])$. Also, in [6], the authors introduced the concept of cone $b_{2}$ -metric space over Banach algebras which generalizes the notions of $b_{2}$-metric space and cone metric spaces over Banach algebra. There are some very recent references (such as $[7,8]$ ), where it is shown that the fixed point theory continues to provide useful tools for studying problems of Mathematical Physics.
The present paper is organized as follows. In Section 2, we recall some definitions. In Section 3, we generalize the concepts of a $G_{p_{b}}$-metric space and a CMS over a Banach algebra by introducing a $J$-CMS over a Banach algebra (for short, $J$-CMS over a BA) with some examples. Section 4 is devoted to define generalized contractive and expansive maps. Finally, in Section 5 and Section 6, we prove some fixed point theorems for such certain contractive and expansive maps in the framework of a $J$-CMS over a BA. Our work generalizes and extends some interesting results of [9]. Two examples and an application are given to verify the strength of our main results.

\section{Preliminaries}

We start with some known concepts. Denote by $Y$ a real Banach algebra (for short, BA). From now on, assume that there is a unit element $e \in Y$. An element $\omega \in Y$ is called invertible if there is $\pi \in Y$ so that $\omega \pi=\pi \omega=e$. We denote by $\omega^{-1}$ the inverse of $\omega$. For more related details, one may check [10]. 
Proposition 1 [10]. Assume that the spectral radius $\mathfrak{S} \mathfrak{R}(\omega)$ of an element $\omega \in Y$ is less than 1 , that is,

$$
\mathfrak{S} \mathfrak{R}(\omega)=\lim _{n \longrightarrow \infty}\left\|\omega^{n}\right\|^{1 / n}=\inf \left\|\omega^{n}\right\|^{1 / n}<1
$$

then $e-\omega$ is invertible, where $e \in Y$ is unit. In addition,

$$
(e-\omega)^{-1}=\sum_{i=0}^{\infty} \omega^{i}
$$

Remark 2 ([11]). If $\mathfrak{S} \Re(\omega)<1$, then $\left\|\omega^{n}\right\| \longrightarrow 0$ as $n \longrightarrow \infty$.

A set $\mathbb{C} \subset Y$ is said to be a cone if

(1) $\mathfrak{E} \neq \varnothing$, closed and $\mathfrak{E} \neq\{\theta\}$

(2) $\alpha \mathfrak{E}+\beta \mathfrak{E} \subset \mathfrak{C}$ for all $\alpha, \beta \geq 0$

(3) $\mathfrak{C}^{2}=\mathfrak{C C} \subset \mathbb{C}$

(4) $(-\mathfrak{S}) \cap \mathfrak{E}=\{\theta\}$,

where $\theta$ is the null of $Y$. The partial ordering on $Y$ is given as $\omega \preccurlyeq \pi$ iff $\pi-\omega \in \mathbb{C}$. We write $\omega \prec \pi$ to indicate $\omega \preccurlyeq \pi$, but $\omega \neq \pi$, while $\omega \ll \pi$ stands for $\pi-\omega \in$ int $\mathbb{E}$ (here, int $\mathbb{E}$ is the interior of $(\mathfrak{E})$.

Definition $3([2,3])$. Let $\mathscr{M}$ be a nonempty set. If $d: \mathscr{M} \times$ $\mathscr{M} \longrightarrow Y$ verifies

(1) $\theta \preccurlyeq d(\omega, \pi)$ for all $\omega, \pi \in \mathscr{M}$ and $d(\omega, \pi)=\theta$ iff $\omega=\pi$

(2) $d(\omega, \pi)=d(\pi, \omega)$ for all $\omega, \pi \in \mathscr{M}$

(3) $d(\omega, \pi) \preccurlyeq d(\omega, \omega)+d(\omega, \pi)$ for all $\omega, \pi, \omega \in \mathscr{M}$

then $d$ is named as a cone metric on $\mathscr{M}$, and $(\mathscr{M}, d)$ is said to be a CMS over the BA $Y$.

Definition 4 ([1]). Let $\mathscr{M}$ be a nonempty set and $s \geq 1$. Assume that the function $G_{p_{b}}: \mathscr{M} \times \mathscr{M} \times \mathscr{M} \longrightarrow \mathbb{R}^{+}$is so that:

$\left(G_{p_{b}} 1\right) \omega=\pi=\omega$ if $G_{p_{b}}(\omega, \pi, \omega)=G_{p_{b}}(\omega, \omega, \omega)=G_{p_{b}}(\pi$, $\pi, \pi)=G_{p_{b}}(\omega, \omega, \omega)$

$\left(G_{p_{b}} 2\right) G_{p_{b}}(\omega, \omega, \omega) \leq G_{p_{b}}(\omega, \omega, \pi) \leq G_{p_{b}}(\omega, \pi, \omega)$ for all $\omega, \pi, \omega \in \mathscr{M}$ with $\omega \neq \pi$

$\left(G_{p_{b}} 3\right) G_{p_{b}}(\omega, \pi, \omega)=G_{p_{b}}(p\{\omega, \pi, \omega\})$, where $p$ is any permutation of $\omega, \pi$ and $\omega$ (symmetry in all three variables)

$\left(G_{p_{b}} 4\right) G_{p_{b}}(\omega, \pi, \omega) \leq s\left[G_{p_{b}}(\omega, a, a)+G_{p_{b}}(a, \pi, \omega)-G_{p_{b}}(a\right.$ $, a, a)]+((1-s) / 3)\left[G_{p_{b}}(\omega, \omega, \omega)+G_{p_{b}}(\pi, \pi, \pi)+G_{p_{b}}(\omega, \omega, \omega\right.$ )] for all $\omega, \pi, \omega, a \in \mathscr{M}$ (rectangle inequality).

Then, $G_{p_{b}}$ is named as a $G_{p_{b}}$-metric and $\left(\mathscr{M}, G_{p_{b}}\right)$ is called a $G_{p_{b}}$-metric space.
Using $\left(G_{p_{b}} 4\right)$ and $s \geq 1$, we have

$G_{p_{b}}(\omega, \pi, \omega) \leq s\left[G_{p_{b}}(\omega, a, a)+G_{p_{b}}(a, \pi, \omega)-G_{p_{b}}(a, a, a)\right]$.

The $G_{p_{b}}$-metric space $G_{p_{b}}$ is called symmetric if $G_{p_{b}}(\omega$, $\omega, \pi)=G_{p_{b}}(\omega, \pi, \pi)$ holds for all $\omega, \pi \in \mathscr{M}$. Otherwise, $G_{p_{b}}$ is an asymmetric $G_{p_{b}}$ - metric.

Example 5 [1]. Let $\mathscr{M}=[0, \infty)$ and let $G_{p_{b}}: \mathscr{M} \times \mathscr{M} \times \mathscr{M}$ $\longrightarrow \mathbb{R}^{+}$be given by $G_{p_{b}}(\omega, \pi, \omega)=[\max \{\omega, \pi, \omega\}]^{p}$ where $p>1$.

Obviously, $\left(\mathscr{M}, G_{p_{b}}\right)$ is a symmetric $G_{p_{b}}$-metric space, which is not a $G$-metric space. In fact, if $\omega=\pi=\omega>0$, then $G_{p_{b}}(\omega, \pi, \omega)=\omega^{p}>0$. It is easy to see that $G_{p_{b}} 1-G_{p_{b}} 4$ are satisfied.

\section{A J-CMS over a Banach Algebra}

Here, we introduce the notion of a $J$-CMS over the BA $Y$, as a generalization of a generalized partial $b$-CMS and a CMS over the BA $Y$.

Definition 6 . Let $\mathscr{M}$ be a nonempty set and $s \geq 1$. Suppose that $J: \mathscr{M} \times \mathscr{M} \times \mathscr{M} \longrightarrow Y$ so that:

$\left(\mathrm{J}_{1}\right) \omega=\pi=\operatorname{\omega if} J(\omega, \pi, \omega)=J(\omega, \omega, \omega)=J(\pi, \pi, \pi)=J(\omega$ , $\omega, \omega)$

$\left(\mathrm{J}_{2}\right) J(\omega, \omega, \omega) \preccurlyeq J(\omega, \omega, \pi) \preccurlyeq J(\omega, \pi, \omega)$ for all $\omega, \pi, \omega \in \mathscr{M}$ with $\varrho \neq \pi$

$\left(J_{3}\right) J(\omega, \pi, \omega)=J(p\{\omega, \pi, \omega\})$, where $p$ is any permutation of $\omega, \pi$ and $\omega$ (symmetry in all three variables)

$\left(\mathrm{J}_{4}\right) \quad J(\omega, \pi, \omega) \leqslant s[J(\omega, a, a)+J(a, \pi, \omega)-J(a, a, a)]+((1$ $-s) / 3)[J(\omega, \omega, \omega)+J(\pi, \pi, \pi)+J(\omega, \omega, \omega)]$ for all $\omega, \pi, \omega, a$ $\in \mathscr{M}$ (rectangle inequality).

Then, $J$ is called a $J$-cone metric and $(\mathscr{M}, J)$ is called a $J$ -CMS over the BA $Y$.

Since $s \geq 1$, from $\left(J_{4}\right)$, we have

$$
J(\omega, \pi, \omega) \preccurlyeq s[J(\omega, a, a)+J(a, \pi, \omega)-J(a, a, a)] .
$$

The $J$-CMS $J$ is said to be symmetric if $J(\omega, \omega, \pi)=J(\omega$ $, \pi, \pi)$ holds for all $\omega, \pi \in \mathscr{M}$. Otherwise, $J$ is asymmetric.

We now present some examples.

Example 7. Let $Y=C_{R}^{1}[0, \infty)$ which is endowed with the norm $\|\omega\|=\|\omega\|_{\infty}+\left\|\omega^{\prime}\right\|_{\infty}$. Under the pointwise multiplication, $Y$ is a real Banach algebra with unit $e=1$. Let $\mathfrak{E}=\{(\omega$, $\tau) \in Y: \omega(t), \tau(t) \geq 0 \forall t \in[0, \infty)\}$. Moreover, $P$ is not normal (see [12]).

Let $G_{p_{b}}:[0, \infty)^{3} \longrightarrow \mathbb{R}^{+}$be given by $G_{p_{b}}(\omega, \pi, \omega)=$ $[\max \{\omega, \pi, \omega\}]^{p}$ where $p \geq 1$. Let $J: \mathscr{M} \times \mathscr{M} \times \mathscr{M} \longrightarrow Y^{2}$ be given by 


$$
J(\omega, \pi, \omega)(t)=\left(G_{p_{b}}(\omega, \pi, \omega), \alpha G_{p_{b}}(\omega, \pi, \omega)\right) e^{t}
$$

Obviously, $\left(\mathscr{M}, G_{p_{b}}\right)$ is not a $G$-metric space. But $(\mathscr{M}, J)$ is a $J$-CMS over the BA $\Upsilon$. Indeed, if $\omega=\pi=\omega>0$, then $J(\omega, \pi, \omega)(t)=\left(\omega^{p}, \alpha \omega^{p}\right) e^{t}>\theta$. Hence, $\left(J_{1}\right)-\left(J_{3}\right)$ are satisfied. Now, we show that $\left(J_{4}\right)$ holds. For all $\omega, \pi, \omega, a \in \mathscr{M}$, we have

$$
\begin{aligned}
{[\max \{\omega, \pi, \omega\}]^{p} e^{t} \leqslant s } & \left([\max \{\omega, a\}]^{p}+[\max \{a, \pi, \omega\}]^{p}-a^{p}\right) e^{t} \\
+ & \frac{(1-s)}{3}\left(\omega^{p}+\pi^{p}+\omega^{p}\right) e^{t},
\end{aligned}
$$

(by Example 12 in [1]). Therefore,

$$
\begin{aligned}
J(\omega, \pi, \omega) \leqslant s[ & J(\omega, a, a)+J(a, \pi, \omega)-J(a, a, a)] \\
& +((1-s) / 3)[J(\omega, \omega, \omega)+J(\pi, \pi, \pi)+J(\omega, \omega, \omega)],
\end{aligned}
$$

for all $\omega, \pi, \omega, a \in \mathscr{M}$ (rectangle inequality).

The following examples show that a $J$-CMS over a BA need not be a $G_{b}$-CMS ([13]).

Example 8. Let $\mathscr{M}=[0,1]$ and $Y$ be the set of all real-valued continuously differentiable functions on $\mathscr{M}$ with the norm $\|\omega\|=\|\omega\|_{\infty}+\left\|\omega^{\prime}\right\|_{\infty}$. Define the multiplication in the usual way. Let $\mathfrak{E}=\{\omega \in Y: \omega(t) \geq 0, t \in \mathscr{M}\}$. Clearly, $\mathfrak{E}$ is a nonnormal cone and $Y$ is a BA with a unit $e=1$. Consider $J: \mathscr{M} \times \mathscr{M} \times \mathscr{M} \longrightarrow Y$ as

$J(\omega, \pi, \omega)(t)=\left(\left(\max \{\omega, \pi\}^{2}\right)+\left(\max \{\pi, \omega\}^{2}\right)+\left(\max \{\omega, \omega\}^{2}\right)\right) e^{t}$,

for all $\omega, \pi, \omega \in \mathscr{M}$. Thus, $(\mathscr{M}, J)$ is a $J$-CMS over the BA $Y$, but it is not a $G_{b}$-CMS since $J(\omega, \omega, \omega)(t)=3 \omega^{2} e^{t} \neq \theta$.

Example 9. Let $Y=C_{\mathbb{R}}^{1}[0,1]$ and consider a norm on $Y$ as $\|$ $\omega\|=\| \omega\left\|_{\infty}+\right\| \omega^{\prime} \|_{\infty}$ for all $\omega \in Y$. Let the multiplication on $Y$ be the pointwise multiplication. Then, $Y$ is a real unit BA with unit $e=1$. Set $\mathbb{E}=\{\omega \in Y: \omega \geq 0\}$ which is a cone in $Y$. Let $\mathscr{M}=[0, \infty)$ and $a>0$ be any constant. Consider $J: \mathscr{M} \times$ $\mathscr{M} \times \mathscr{M} \longrightarrow Y$ as

$$
J(\omega, \pi, \omega)(t)=\left([\max \{\omega, \pi, \omega\}]^{2}+a\right) e^{t},
$$

for all $\omega, \pi, \omega \in \mathscr{M}$. This $(\mathscr{M}, J)$ is a $J$-CMS over the BA $Y$, but it is not a $G_{b}$-CMS since $J(\omega, \omega, \omega)(t)=\left(\omega^{2}+a\right) e^{t} \neq \theta$.

Example 10. Let $Y=C[a, b]$ be the set of all continuous functions on $[a, b]$ with the norm $\|\omega\|=\|\omega\|_{\infty}+\left\|\omega^{\prime}\right\|_{\infty}$. Taking the usual multiplication, then $Y$ is a BA with a unit 1 . Set $\mathfrak{E}$ $=\{\omega \in Y: \omega(t) \geq 0, t \in[a, b]\}$ and $\mathscr{M}=\mathbb{R}^{+}$. Consider $J: \mathscr{M}$ $\times \mathscr{M} \times \mathscr{M} \longrightarrow Y$ by

$$
J(\omega, \pi, \omega)(t)=\left([\max \{\omega, \pi, \omega\}]^{2}+|\omega-\pi|^{2}+|\pi-\omega|^{2}+|\omega-\omega|^{2}\right) e^{t},
$$

for all $\omega, \pi, \omega \in \mathscr{M}$. Then, $(\mathscr{M}, J)$ is a $J$-CMS over the BA $Y$. But it is not a $G_{b}$-CMS, because $J(\omega, \omega, \omega)(t)=\omega^{2} e^{t} \neq \theta$.

In the following, $(\mathscr{M}, J)$ is assumed to be a $J$-CMS over the BA $Y$.

Lemma 11. (a) If $J(\omega, \pi, \omega)=\theta$, then $\omega=\pi=\omega$.

(b) If $\omega \neq \pi$, then $J(\omega, \pi, \pi)>\theta$.

Definition 12. For an $\omega \in \mathscr{M}$ and $c>\theta$, the $J$-ball with center $\omega$ and radius $c>\theta$ is

$$
B_{J}(\omega, c)=\{\pi \in \mathscr{M}: J(\omega, \omega, \pi) \ll J(\omega, \omega, \omega)+c\} .
$$

The $\theta$-Cauchyness and convergence are given as follows.

Definition 13. A sequence $\left\{\omega_{n}\right\}$ in $(\mathscr{M}, J)$ converges to $\omega \in \mathscr{M}$ , whenever for each $c \gg \theta$, there is an integer $N$ so that $J\left(\omega_{n}\right.$ $, \omega, \omega) \ll c$ for all $n \geq N$.

Definition 14. A sequence $\left\{\omega_{n}\right\}$ in $\mathscr{M}$ is called a $\theta$-Cauchy sequence in $(\mathscr{M}, J)$ if for every $c \gg \theta$ there is $n_{0} \in N$ such that $J\left(\omega_{n}, \omega_{m}, \omega_{m}\right) \ll c$ for all $n, m \geq n_{0}$.

Definition $15 . \mathscr{M}$ is said to be $\theta$-complete if every $\theta$-Cauchy sequence $\left\{\omega_{n}\right\}$ in $(\mathscr{M}, J)$ is convergent to $\omega \in \mathscr{M}$ so that $J(\omega, \omega, \omega)=\theta$.

Definition 16. Let $\left(\mathscr{M}^{\prime}, J^{\prime}\right)$ and $\left(\mathscr{M}^{\prime}, J^{\prime}\right)$ be two $J$-CMS over the BA $Y$. Then, a function $f: \mathscr{M} \longrightarrow \mathscr{M}^{\prime}$ is called continuous at $\omega \in \mathscr{M}$ iff for each convergent sequence $\left\{\omega_{n}\right\}$ to $\omega$, we have $\left\{f \omega_{n}\right\}$ is convergent to $f(\omega)$.

\section{Generalized Contractive and Expansive Maps}

In this section, we consider contractive and expansive maps in a J-CMS over a BA. Some examples are also presented.

Definiton 17. Let $(\mathscr{M}, J)$ be a $J$-CMS over the BA $Y$ and $\mathfrak{E}$ be a cone in $Y$. A map $\chi: \mathscr{M} \longrightarrow \mathscr{M}$ is named as a generalized contractive mapping if there is $k \in \mathfrak{C}$ (with $\mathfrak{S} \mathfrak{R}(k)<1$ ) so that for all $\omega, \pi, \omega \in \mathscr{M}$,

$$
J(\chi \omega, \chi \pi, \chi \omega) \preccurlyeq k J(\omega, \pi, \omega) .
$$

Example 18. Let $Y$ be a BA and $\mathfrak{C}$ be a cone (as in Example 8) and let $\mathscr{M}=\mathbb{R}^{+}$. Define a mapping $J: \mathscr{M} \times \mathscr{M} \times \mathscr{M} \longrightarrow Y$ by

$$
J(\omega, \pi, \omega)(t)=\left([\max \{\omega, \pi, \omega\}]^{2}+|\omega-\pi|^{2}+|\pi-\omega|^{2}+|\omega-\omega|^{2}\right) e^{t},
$$

for all $\omega, \pi, \omega \in \mathscr{M}$. Then, $(\mathscr{M}, J)$ is a $J$-CMS over the BA $Y$. Take $\chi: \mathscr{M} \longrightarrow \mathscr{M}$ by $\chi(\omega)=\ln (1+(\omega / 2))$. In view of $\ln (1+u) \leq u$ for each $u \geq 0$, we have for all $\omega, \pi, \omega \in \mathscr{M}$, 


$$
\begin{aligned}
J(\chi \omega, \chi \pi, \chi \omega)(t)= & \left(\left[\max \left\{\ln \left(1+\frac{\omega}{2}\right), \ln \left(1+\frac{\pi}{2}\right), \ln \left(1+\frac{\omega}{2}\right)\right\}\right]^{2}\right. \\
& +\left|\ln \left(1+\frac{\omega}{2}\right)-\ln \left(1+\frac{\pi}{2}\right)\right|^{2}+\mid \ln \left(1+\frac{\pi}{2}\right) \\
& \left.-\left.\ln \left(1+\frac{\omega}{2}\right)\right|^{2}+\left|\ln \left(1+\frac{\omega}{2}\right)-\ln \left(1+\frac{\omega}{2}\right)\right|^{2}\right) e^{t} \\
\preccurlyeq & \left(\left[\max \left\{\frac{\omega}{2}, \frac{\pi}{2}, \frac{\omega}{2}\right\}\right]^{2}+\left|\frac{\omega}{2}-\frac{\pi}{2}\right|^{2}+\left|\frac{\pi}{2}-\frac{\omega}{2}\right|^{2}+\left|\frac{\omega}{2}-\frac{\omega}{2}\right|^{2}\right) e^{t} \\
\leqslant & \frac{1}{4}\left([\max \{\omega, \pi, \omega\}]^{2}+|\omega-\pi|^{2}+|\pi-\Phi|^{2}+|\omega-\omega|^{2}\right) e^{t} \\
= & \frac{1}{4} J(\omega, \pi, \varpi)(t),
\end{aligned}
$$

where $k=1 / 4$. Obviously, $\chi$ is a generalized contractive map on $\mathscr{M}$.

Definition 19. Let $(\mathscr{M}, J)$ be a $J$-CMS over the BA $Y$ and $\mathfrak{C}$ be a cone in $Y$. A map $\chi: \mathscr{M} \longrightarrow \mathscr{M}$ is said to be an expansive mapping if for all $\omega, \pi, \omega \in \mathscr{M}$,

$$
J(\chi \omega, \chi \pi, \chi \omega) \geqslant k J(\omega, \pi, \omega),
$$

where $k, k^{-1} \in \mathfrak{C}$ and are called generalized contractive constants with $\mathfrak{S} \mathfrak{R}\left(k^{-1}\right)<1$.

Example 20. Let $Y$ be a BA and $\mathfrak{c}$ be a cone (as given in Example 9) and let $\mathscr{M}=\mathbb{R}^{+}$. As in Example $18,(\mathscr{M}, J)$ is a $J$-CMS over the BA Y. Define $\chi \omega=3 \omega+\omega^{2}$ for all $\omega$.

$$
\begin{aligned}
J(\chi \omega, \chi \pi, \chi \omega)(t)= & {\left[\left(\left[\max \left\{3 \omega+\omega^{2}, 3 \pi+\pi^{2}, 3 \omega+\omega^{2}\right\}\right]^{2}\right.\right.} \\
& +\left|\left(3 \omega+\omega^{2}\right)-\left(3 \pi+\pi^{2}\right)\right|^{2}+\left|\left(3 \pi+\pi^{2}\right)-\left(3 \omega+\omega^{2}\right)\right|^{2} \\
& \left.\left.+\left|\left(3 \omega+\omega^{2}\right)-\left(3 \omega+\omega^{2}\right)\right|^{2}\right)\right] e^{t} \\
= & \left(\left[\max \left\{3 \omega+\omega^{2}, 3 \pi+\pi^{2}, 3 \omega+\omega^{2}\right\}\right]^{2}\right. \\
& +|3(\omega-\pi)+(\omega-\pi)(\omega+\pi)|^{2} \\
& +|3(\pi-\omega)+(\pi-\omega)(\pi+\omega)|^{2} \\
& \left.+|3(\omega-\omega)+(\omega-\omega)(\omega+\omega)|^{2}\right) e^{t} \\
= & \left(\left[\max \left\{3 \omega+\omega \omega^{2}, 3 \pi+\pi^{2}, 3 \omega+\omega^{2}\right\}\right]^{2}\right. \\
& +|\omega-\pi|^{2}|3+(\omega+\pi)|^{2}+|\pi-\omega|^{2}|3+(\pi+\omega)|^{2} \\
& \left.+|\omega-\omega|^{2}|3+(\omega+\omega)|^{2}\right) e^{t} \\
\succcurlyeq & \left([\max \{3 \omega, 3 \pi, 3 \omega\}]^{2}+9|\omega-\pi|^{2}\right. \\
& \left.+9|\pi-\omega|^{2}+9|\omega-\omega|^{2}\right) e^{t} \\
\succcurlyeq & 9\left([\max \{\omega, \pi, \omega\}]^{2}+|\omega-\pi|^{2}+|\pi-\omega|^{2}+|\omega-\omega|^{2}\right) e^{t} \\
= & 9 J(\omega, \pi, \omega)(t),
\end{aligned}
$$

where $k=9$. Clearly, $\chi$ is an expansive map in $\mathscr{M}$.

Definition 21 ([14]). Let $\mathfrak{5}$ be a solid cone in a Banach space $\mathscr{E}$. A sequence $\left\{u_{n}\right\} \subset \mathbb{C}$ is said to be a $c$-sequence if for every $c \gg \theta$ there is an integer $N$ so that $u_{n} \ll c$ for all $n>N$.
Lemma 22 ([5]). If $\mathscr{E}$ is a real Banach space with a solid cone $\mathfrak{V}$, then $\left\{u_{n}\right\}$ is a c-sequence whenever $\left\{u_{n}\right\} \subset \mathbb{\mathfrak { C }}$ be a sequence with $\left\|u_{n}\right\| \longrightarrow 0(\infty)$.

Lemma 23 ([10]). Let $Y$ be a BA with a unite and $k \in Y$. Then, $\lim \left\|k^{n}\right\|^{1 / n}$ exists, and the spectral radius $\mathfrak{S} \mathfrak{R}(k)$ verifies

$$
\mathfrak{S} \mathfrak{R}(k)=\lim \left\|k^{n}\right\|^{1 / n}=\inf _{n}\left\|k^{n}\right\|^{1 / n} .
$$

Then, $(\zeta e-k)$ is invertible in $Y$ provided that $\mathfrak{S} \mathfrak{R}(k)$ $<|\zeta|$. Moreover,

$$
(\zeta e-k)^{-1}=\sum_{i=0}^{\infty} \frac{k^{i}}{\zeta^{i+1}}
$$

where the constant $\zeta$ is complex.

Lemma 24 ([10]). Let $Y$ be a $B A$ with a unit e and $\vartheta, \omega \in Y$. Then,

$\mathfrak{S R}(\vartheta+\varpi) \leq \mathfrak{S R}(\vartheta)+\mathfrak{S} \Re(\omega), \mathfrak{S} \Re(\vartheta \omega) \leq \mathfrak{S} R(\vartheta) \widetilde{R}(\omega)$,

provided that $\vartheta$ commutes with $\varpi$.

Lemma 25 ([12]). Let $\mathscr{E}$ be a real Banach space with a solid cone 5 . Then,

(1) $\vartheta \ll \kappa$, if $\vartheta \leq \omega \ll \kappa$

(2) $\vartheta=\theta$, if $\vartheta \ll \kappa$ for every $\kappa \gg \theta$

Lemma 26 ([11]). Let $\mathbb{5}$ be a solid cone in the BA $Y$ and suppose that $k \in \mathbb{C}$. Then, $\left\{k u_{n}\right\}$ is a $c$-sequence provided that $\left\{u_{n}\right\}$ be a c-sequence in $\mathfrak{c}$.

Lemma 27 ([5]). Let $Y$ be a BA with a unit e and let $k \in Y$. Then,

$$
\mathfrak{S} \mathfrak{R}\left((\zeta e-k)^{-1}\right) \leq \frac{1}{|\zeta|-\mathfrak{S} \mathfrak{R}(k)},
$$

where $\zeta$ is a complex constant with $\mathfrak{\Im} \mathfrak{R}(k)<|\zeta|$.

Lemma 28 ([5]). Let $\mathfrak{c}$ be a solid cone in the BA Y with a unit $e$ and let $\vartheta, k, l \in \mathbb{E}$ so that $l \leqslant k$ and $\vartheta \leqslant l \vartheta$. Then, $\vartheta=\theta$ provided that $\mathfrak{S} \Re(k)<1$.

In this paper, we prove some fixed-point theorems for generalized contractive and expansive maps in the setting of a $J$-CMS over a BA.

\section{Main Results}

Theorem 29. Let $(\mathscr{M}, J)$ be a $\theta$-complete symmetric J-CMS over the $B A Y$ and $\chi: \mathscr{M} \longrightarrow \mathscr{M}$ be a mapping so that for all $\omega, \pi \in \mathscr{M}$ 


$$
J(\chi \omega, \chi \pi, \chi \pi) \leqslant k J(\omega, \pi, \pi),
$$

where $\mathfrak{S} \mathfrak{R}(k)<1 / s$. Then, $\chi$ admits a unique fixed point.

Proof. Let $\omega_{0}$ be an arbitrary point in $\mathscr{M}$. Define a sequence $\left\{\omega_{n}\right\}$ in $\mathscr{M}$ by $\omega_{n}=\chi^{n} \omega_{0}$. By (21), we have

$$
J\left(\omega_{n}, \omega_{n+1}, \omega_{n+1}\right)=J\left(\chi \omega_{n-1}, \chi \omega_{n}, \chi \omega_{n}\right) \preccurlyeq k J\left(\omega_{n-1}, \omega_{n}, \omega_{n}\right)
$$

Continuing in the same argument, we will get

$$
J\left(\omega_{n}, \omega_{n+1}, \omega_{n+1}\right) \preccurlyeq k^{n} J\left(\omega_{0}, \omega_{1}, \omega_{1}\right) .
$$

Moreover, for all $n, m \in N ; n<m$, we have

$$
\begin{aligned}
& J\left(\omega_{n}, \omega_{m}, \omega_{m}\right) \preccurlyeq s\left[J\left(\omega_{n}, \omega_{n+1}, \omega_{n+1}\right)+J\left(\omega_{n+1}, \omega_{m}, \omega_{m}\right)-J\left(\omega_{n+1}, \omega_{n+1}, \omega_{n+1}\right)\right] \\
& \preccurlyeq s J\left(\omega_{n}, \omega_{n+1}, \omega_{n+1}\right)+s J\left(\omega_{n+1}, \omega_{m}, \omega_{m}\right) \\
& \preccurlyeq s J\left(\omega_{n}, \omega_{n+1}, \omega_{n+1}\right)+s^{2}\left[J\left(\omega_{n+1}, \omega_{n+2}, \omega_{n+2}\right)\right. \\
&\left.+J\left(\omega_{n+2}, \omega_{m}, \omega_{m}\right)-J\left(\omega_{n+2}, \omega_{n+2}, \omega_{n+2}\right)\right] \\
& \preccurlyeq s\left(\omega_{n}, \omega_{n+1}, \omega_{n+1}\right)+s^{2} J\left(\omega_{n+1}, \omega_{n+2}, \omega_{n+2}\right)+s^{2} J\left(\omega_{n+2}, \omega_{m}, \omega_{m}\right) \\
& \vdots \leqslant s J\left(\omega_{n}, \omega_{n+1}, \omega_{n+1}\right)+s^{2} J\left(\omega_{n+1}, \omega_{n+2}, \omega_{n+2}\right)+\cdots \\
&+s^{m-n} J\left(\omega_{m-1}, \omega_{m}, \omega_{m}\right) \leqslant s k^{n} J\left(\omega_{0}, \omega_{1}, \omega_{1}\right) \\
&+s^{2} k^{n+1} J\left(\omega_{0}, \omega_{1}, \omega_{1}\right)+\cdots+s^{m-n} k^{m-1} J\left(\omega_{0}, \omega_{1}, \omega_{1}\right) \\
&= s k^{n}\left[e+s k+(s k)^{2}+\cdots+(s k)^{m-n-1}\right] J\left(\omega_{0}, \omega_{1}, \omega_{1}\right) \\
& \preccurlyeq s k^{n}\left(\sum_{i=0}^{\infty}(s k)^{i}\right) J\left(\omega_{0}, \omega_{1}, \omega_{1}\right)=(e-s k)^{-1} s k^{n} J\left(\omega_{0}, \omega_{1}, \omega_{1}\right) .
\end{aligned}
$$

In view of Remark 2, $\left\|s k^{n} J\left(\omega_{0}, \omega_{1}, \omega_{1}\right)\right\| \leqslant\left\|k^{n}\right\| \| J\left(\omega_{0}, \omega_{1}\right.$, $\left.\omega_{1}\right) \| \longrightarrow 0(\infty)$, by Lemma 22 , we have $\left\{s k^{n} J\left(\omega_{0}, \omega_{1}, \omega_{1}\right)\right\}$ is a $c$-sequence. Using Lemma 25 and Lemma $26,\left\{\omega_{n}\right\}$ is a $\theta$-Cauchy sequence in $\mathscr{M}$. By the $\theta$-completeness of $\mathscr{M}$, there is $\rho \in \mathscr{M}$ so that

$$
\lim J\left(\omega_{n}, \varrho, \varrho\right)=\lim _{n, m \longrightarrow \infty} J\left(\omega_{n}, \omega_{m}, \omega_{m}\right)=J(\varrho, \varrho, \varrho)=\theta
$$

Furthermore, one has

$$
\begin{aligned}
J(\varrho, \chi \varrho, \chi \varrho) & \leqslant s J\left(\varrho, \chi \omega_{n}, \chi \omega_{n}\right)+s J\left(\chi \omega_{n}, \chi \varrho, \chi \varrho\right)-s J\left(\chi \omega_{n}, \chi \omega_{n}, \chi \omega_{n}\right) \\
& \leqslant s J\left(\varrho, \chi \omega_{n}, \chi \omega_{n}\right)+s J\left(\chi \omega_{n}, \chi \varrho, \chi \varrho\right) \\
& \leqslant s J\left(\varrho, \omega_{n+1}, \omega_{n+1}\right)+s k J\left(\omega_{n}, \omega_{n+1}, \varrho\right) .
\end{aligned}
$$

Hence, $\left\{J\left(\varrho, \omega_{n+1}, \omega_{n+1}\right)\right\}$ and $\left\{J\left(\omega_{n}, \omega_{n+1}, \varrho\right)\right\}$ are $c$ -sequences, then by using Lemma 25 and Lemma 26, we get that $\chi \mathrm{Q}=\mathrm{Q}$. Thus, $\mathrm{Q}$ is a fixed point of $\chi$. If $\chi \zeta=\zeta$, then one writes

$$
J(\varrho, \varrho, \zeta)=J(\chi \varrho, \chi \varrho, \chi \zeta)=k J(\varrho, \varrho, \zeta)
$$

That is,

$$
(e-k) J(\varrho, \varrho, \zeta) \preccurlyeq \theta
$$

The multiplication by

$$
(e-k)^{-1}=\sum_{i=0}^{\infty} k^{i} \geq 0
$$

yields that $J(\varrho, \varrho, \zeta) \preccurlyeq \theta$. Thus, $J(\varrho, \varrho, \zeta)=\theta$, so $\varrho=\zeta$, a contradiction. Hence, the fixed point is unique.

Corollary 30. Let $(\mathscr{M}, J)$ be a $\theta$-complete symmetric J-CMS over the $B A Y$ and $\chi: M \longrightarrow M$ be a mapping so that for all $\omega, \pi, \omega \in \mathscr{M}$

$$
J(\chi \omega, \chi \pi, \chi \omega) \preccurlyeq a J(\omega, \chi \omega, \omega)+b J(\omega, \chi \omega, \pi),
$$

where $\mathfrak{S} \mathfrak{R}(a+b)<1 /$ s. Then, $\chi$ has a unique fixed point.

Proof. In view of the proof of Theorem 29, we get the required result.

Theorem 31. Let $(\mathscr{M}, J)$ be a $\theta$-complete symmetric J-CMS over the $B A Y$ and $\chi: \mathscr{M} \longrightarrow \mathscr{M}$ be a mapping so that for all $\omega, \pi \in \mathscr{M}$,

$$
\begin{aligned}
J\left(\chi \omega, \chi \pi, \chi^{2} \pi\right) \leqslant & J\left(\omega, \chi \omega, \chi^{2} \omega\right)+b J\left(\pi, \chi \pi, \chi^{2} \pi\right) \\
& +c J(\omega, \chi \omega, \chi \pi)+d J\left(\pi, \chi \pi, \chi^{3} \omega\right),
\end{aligned}
$$

where $s \mathfrak{S} \mathfrak{R}(a+c)+\mathfrak{S} \mathfrak{R}(b+d)<1$ and $s^{2} \mathfrak{S} \mathfrak{R}(c+d)+$ $\mathfrak{S}(b s)<1$. Then, $\chi$ admits a unique fixed point.

Proof. Take $\omega_{0} \in \mathscr{M}$. We construct $\left\{\omega_{n}\right\}$ in $\mathscr{M}$ so that

$$
\omega_{n+1}=\chi \omega_{n}, \text { for all } n=0,1,2, \cdots
$$

Notice that if $\omega_{n^{\prime}}=\omega_{n^{\prime}+1}$ for some integer $n^{\prime}$, then there is a fixed point. Suppose that $\omega_{n} \neq \omega_{n+1}$ for all $n \in N$. That is,

$$
J\left(\omega_{n}, \omega_{n+1}, \omega_{n+2}\right)>\theta
$$

From (31), with $\omega=\omega_{n-1}$ and $\pi=\omega_{n}$, we have

$$
\begin{array}{rl}
J\left(\chi \omega_{n-1}, \chi \omega_{n}, \chi^{2} \omega_{n}\right) \leqslant a & J\left(\omega_{n-1}, \chi \omega_{n-1}, \chi^{2} \omega_{n-1}\right)+b J\left(\omega_{n}, \chi \omega_{n}, \chi^{2} \omega_{n}\right) \\
+ & c J\left(\omega_{n-1}, \chi \omega_{n-1}, \chi \omega_{n}\right)+d J\left(\omega_{n}, \chi \omega_{n}, \chi^{3} \omega_{n-1}\right),
\end{array}
$$

which implies that

$$
\begin{array}{rl}
J\left(\omega_{n}, \omega_{n+1}, \omega_{n+2}\right) \leqslant a & J\left(\omega_{n-1}, \omega_{n}, \omega_{n+1}\right)+b J\left(\omega_{n}, \omega_{n+1}, \omega_{n+2}\right) \\
& +c J\left(\omega_{n-1}, \omega_{n}, \omega_{n+1}\right)+d J\left(\omega_{n}, \omega_{n+1}, \omega_{n+2}\right), \\
= & (a+c) J\left(\omega_{n-1}, \omega_{n}, \omega_{n+1}\right)+(b+d) J\left(\omega_{n}, \omega_{n+1}, \omega_{n+2}\right) .
\end{array}
$$

Thus,

$$
(e-b-d) J\left(\omega_{n}, \omega_{n+1}, \omega_{n+2}\right) \preccurlyeq(a+c) J\left(\omega_{n-1}, \omega_{n}, \omega_{n+1}\right) .
$$


Since $s \mathbb{S} \mathfrak{R}(a+c)+\mathfrak{S} \mathfrak{R}(b+d)<1$ leads to $\mathfrak{S} \mathfrak{R}(b+d)$ $<1$, by Lemma $23,(e-b-d)$ is invertible. So

$$
J\left(\omega_{n}, \omega_{n+1}, \omega_{n+2}\right) \leqslant(e-b-d)^{-1}(a+c) J\left(\omega_{n-1}, \omega_{n}, \omega_{n+1}\right) .
$$

Put $k=(e-b-d)^{-1}(a+c)$. Hence,

$$
J\left(\omega_{n}, \omega_{n+1}, \omega_{n+2}\right) \leqslant k J\left(\omega_{n-1}, \omega_{n}, \omega_{n+1}\right) \leqslant \cdots \leqslant k^{n} J\left(\omega_{0}, \omega_{1}, \omega_{2}\right) .
$$

By Lemma 24 and Lemma 27, we have

$$
\begin{aligned}
\mathfrak{S} \mathfrak{R}(k) & =\mathfrak{S} \mathfrak{R}\left[(e-b-d)^{-1}(a+c)\right] \\
& \leq \mathfrak{S} \mathfrak{R}\left((e-b-d)^{-1}\right) \mathfrak{S} \mathfrak{R}(a+c) \\
& \leq \frac{\mathfrak{S} \mathfrak{R}(a+c)}{1-\mathfrak{S} \mathfrak{R}(b+d)}<\frac{1}{s} .
\end{aligned}
$$
$\left(J_{2}\right)$,

Thus, $(e-s k)$ is invertible, so $\left\|(s k)^{n}\right\| \longrightarrow 0(\infty)$. Due to

$$
J\left(\omega_{n}, \omega_{n}, \omega_{n+1}\right) \preccurlyeq J\left(\omega_{n}, \omega_{n+1}, \omega_{n+2}\right),
$$

with $\omega_{n} \neq \omega_{n+1}$, and by the symmetry property

$$
J\left(\omega_{n+1}, \omega_{n+1}, \omega_{n}\right)=J\left(\omega_{n+1}, \omega_{n}, \omega_{n}\right)=J\left(\omega_{n}, \omega_{n}, \omega_{n+1}\right) .
$$

Then, by (38) and (41), we have $J\left(\omega_{n+1}, \omega_{n+1}, \omega_{n}\right) \leqslant k^{n}$ $J\left(\omega_{0}, \omega_{1}, \omega_{2}\right)$. Moreover, for all $n, m \in N ; n<m$, we have

$$
\begin{aligned}
J\left(\omega_{n}, \omega_{m}, \omega_{m}\right) \preccurlyeq & s\left[\left(\omega_{n}, \omega_{n+1}, \omega_{n+1}\right)+J\left(\omega_{n+1}, \omega_{m}, \omega_{m}\right)-J\left(\omega_{n+1}, \omega_{n+1}, \omega_{n+1}\right)\right] \\
\preccurlyeq & s J\left(\omega_{n}, \omega_{n+1}, \omega_{n+1}\right)+s J\left(\omega_{n+1}, \omega_{m}, \omega_{m}\right) \\
\preccurlyeq & s J\left(\omega_{n}, \omega_{n+1}, \omega_{n+1}\right)+s^{2}\left[J\left(\omega_{n+1}, \omega_{n+2}, \omega_{n+2}\right)+J\left(\omega_{n+2}, \omega_{m}, \omega_{m}\right)\right. \\
& \left.-J\left(\omega_{n+2}, \omega_{n+2}, \omega_{n+2}\right)\right] \preccurlyeq s J\left(\omega_{n}, \omega_{n+1}, \omega_{n+1}\right) \\
& +s^{2} J\left(\omega_{n+1}, \omega_{n+2}, \omega_{n+2}\right)+s^{2} J\left(\omega_{n+2}, \omega_{m}, \omega_{m}\right) \\
& \vdots \leqslant s J\left(\omega_{n}, \omega_{n+1}, \omega_{n+1}\right)+s^{2} J\left(\omega_{n+1}, \omega_{n+2}, \omega_{n+2}\right)+\cdots+s^{m-n} J\left(\omega_{m-1}, \omega_{m}, \omega_{m}\right) \\
\preccurlyeq & s k^{n} J\left(\omega_{0}, \omega_{1}, \omega_{2}\right)+s^{2} k^{n+1} J\left(\omega_{0}, \omega_{1}, \omega_{2}\right)+\cdots+s^{m-n} k^{m-1} J\left(\omega_{0}, \omega_{1}, \omega_{2}\right) \\
= & s k^{n}\left[e+s k+(s k)^{2}+\cdots+(s k)^{m-n-1}\right] J\left(\omega_{0}, \omega_{1}, \omega_{2}\right) \\
\preccurlyeq & s k^{n}\left(\sum_{i=0}^{\infty}(s k)^{i}\right) J\left(\omega_{0}, \omega_{1}, \omega_{2}\right)=s k^{n}(e-s k)^{-1} J\left(\omega_{0}, \omega_{1}, \omega_{2}\right) .
\end{aligned}
$$

In view of Remark 2, $\left\|s k^{n} J\left(\omega_{0}, \omega_{1}, \omega_{2}\right)\right\| \leqslant\left\|s k^{n}\right\| \| J\left(\omega_{0}\right.$, $\left.\omega_{1}, \omega_{2}\right) \| \longrightarrow 0(\infty)$; by Lemma 22 , we have $\left\{s k^{n} J\left(\omega_{0}, \omega_{1}\right.\right.$, $\left.\left.\omega_{2}\right)\right\}$ is a $c$-sequence. Next, by using Lemma 25 and Lemma $26, \omega_{n}$ is a $\theta$-Cauchy sequence in $\mathscr{M}$. By the $\theta$ -completeness of $\mathscr{M}$, there is $\emptyset \in \mathscr{M}$ so that

$$
\lim J\left(\omega_{n}, \Phi, \Phi\right)=\lim _{n, m \longrightarrow \infty} J\left(\omega_{n}, \omega_{m}, \omega_{m}\right)=J(\omega, \Phi, \emptyset)=\theta
$$

To see that $\omega$ is a fixed point of $\chi$, we have

$$
\begin{aligned}
& J\left(\omega, \chi \omega, \chi^{2} \omega\right) \leqslant s\left[J\left(\omega, \chi \omega_{n}, \chi \omega_{n}\right)+J\left(\chi \omega_{n}, \chi \omega, \chi^{2} \oplus\right)-J\left(\chi \omega_{n}, \chi \omega_{n}, \chi \omega_{n}\right)\right] \\
& =s J\left(\Phi, \chi \omega_{n}, \chi \omega_{n}\right)+s J\left(\chi \omega_{n}, \chi \emptyset, \chi^{2} \oplus\right) \\
& \leqslant s J\left(\omega, \chi \omega_{n}, \chi \omega_{n}\right)+s\left[a J\left(\omega_{n}, \chi \omega_{n}, \chi^{2} \omega_{n}\right)+b J\left(\Phi, \chi \omega, \chi^{2} \oplus\right)\right. \\
& \left.+c J\left(\omega_{n}, \chi \omega_{n}, \chi \omega\right)+d J\left(\Phi, \chi \omega, \chi^{3} \omega_{n}\right)\right] .
\end{aligned}
$$

Then,

$$
\begin{aligned}
& J\left(\varpi, \chi \emptyset, \chi^{2} \varpi\right) \leqslant s J\left(\varrho, \omega_{n+1}, \omega_{n+1}\right)+s\left[a J\left(\omega_{n}, \omega_{n+1}, \omega_{n+2}\right)\right. \\
& +b J\left(\varpi, \chi \emptyset, \chi^{2} \varpi\right)+c J\left(\omega_{n}, \omega_{n+1}, \chi \varpi\right) \\
& \left.+d J\left(\omega, \chi \omega, \omega_{n+3}\right)\right] \\
& \preccurlyeq s b J\left(\omega, \chi \emptyset, \chi^{2} \varpi\right)+c s J(\varpi, \emptyset, \chi \varpi) \\
& +s d J(\varpi, \chi \emptyset, \emptyset),
\end{aligned}
$$

which implies that

$$
(e-b s) J\left(\varpi, \chi \omega, \chi^{2} \varpi\right) \leqslant s(c+d) J(\varpi, \emptyset, \chi \varpi) .
$$

Since $s^{2} \mathfrak{S} \mathfrak{R}(c+d)+\mathfrak{S} \mathfrak{R}(b s)<1$ leads to $\mathfrak{S} \mathfrak{R}(b s)<1$, it follows by Lemma 23 that $(e-b s)$ is invertible. So, $J(\omega$, $\left.\chi \emptyset, \chi^{2} \varpi\right) \preccurlyeq(e-b s)^{-1} s(c+d) J(\emptyset, \emptyset, \chi \omega)$. Put $h=(e-b s)^{-1} s(c$ $+d)$. Then

$$
J\left(\omega, \chi \omega, \chi^{2} \omega\right) \leqslant h J(\omega, \varpi, \chi \omega) .
$$

Note that by Lemma 24 and Lemma 27,

$$
\begin{aligned}
\mathfrak{S} \mathfrak{R}(h) & =\mathfrak{S} \mathfrak{R}\left[(e-b s)^{-1}(s c+s d)\right] \\
& \leq \mathfrak{S} \mathfrak{R}((e-b s))^{-1} \mathfrak{S} \mathfrak{R}(s c+s d) \\
& \leq \frac{\mathfrak{S} \mathfrak{R}(s c+s d)}{1-\mathfrak{S} \mathfrak{R}(b s)}<\frac{1}{s} .
\end{aligned}
$$

In view of (47), there is a arise 2 cases.

Case I. If $\chi ळ=\chi^{2} \varpi$, then

$$
\begin{aligned}
& J(\varpi, \chi \oplus, \chi \emptyset) \leqslant h J(\Phi, \oplus, \chi \varpi) \\
& \leqslant h J(\omega, \chi \omega, \chi \omega) \quad \text { (by symmetry) } \\
& (e-h) J(\varrho, \chi \omega, \chi \omega) \leqslant \theta .
\end{aligned}
$$

We multiply by

$$
(e-h)^{-1}=\sum_{i=0}^{\infty}\left(h^{i}\right) \geq 0,
$$

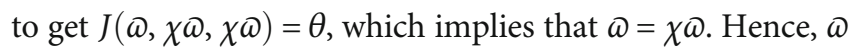
is the fixed point of $\chi$. 
Case II. If $\chi ळ \neq \chi^{2} \varpi$, then

$$
\begin{aligned}
& J\left(\omega, \chi \omega, \chi^{2} \varpi\right) \leqslant h J(\omega, \omega, \chi \varpi) \\
& \leqslant h J(\oplus, \chi \omega, \chi \emptyset) \quad \text { (by symmetry) } \\
& \preccurlyeq J\left(\omega, \chi \omega, \chi^{2} \omega\right) \quad\left(b y J_{2}\right) \\
& (e-h) J\left(\omega, \chi \omega, \chi^{2} \varpi\right) \quad \leq \theta .
\end{aligned}
$$

Again, we multiply by

$$
(e-h)^{-1}=\sum_{i=0}^{\infty}\left(h^{i}\right) \geq 0,
$$

to have $J\left(\varpi, \chi \emptyset, \chi^{2} \varpi\right)=\theta$. So, $\varpi=\chi \emptyset=\chi^{2} \varpi$. Hence, $\varpi$ is a fixed point of $\chi$. Suppose that $w(\neq \emptyset)$ is such that $\chi w=w$. One writes

$$
\begin{aligned}
& J(\omega, \oplus, \varpi)=J(\chi \omega, \chi \omega, \chi \varpi) \\
& =J(\chi \omega, \chi w, \chi w) \text { (by symmetry) } \\
& =J\left(\chi \omega, \chi w, \chi^{2} w\right) \\
& \preccurlyeq a J\left(\varrho, \chi \emptyset, \chi^{2} \varpi\right)+b J\left(w, \chi w, \chi^{2} w\right)+c J(\omega, \chi \emptyset, \chi w)+d J\left(w, \chi w, \chi^{3} \varpi\right) \\
& \preccurlyeq a J(\varpi, \emptyset, \emptyset)+b J(w, w, w)+c J(\Phi, \emptyset, w)+d J(w, w, \varpi) \\
& \leqslant a J(Ф, \emptyset, \emptyset)+b J(w, w, w)+c J(\varpi, \emptyset, w)+d J(w, w, \emptyset)\left[b y\left(J_{2}\right)\right] .
\end{aligned}
$$

Hence,

$$
J(Ф, \emptyset, w) \preccurlyeq(b+c+d) J(\omega, \emptyset, w) .
$$

Since $\mathbb{S} \mathfrak{R}(b+c+d) \leq s^{2} \mathfrak{S} \mathfrak{R}(c+d)+\mathfrak{S} \mathfrak{R}(b s)<1$, by Lemma 28 , we acquire that $J(\emptyset, \emptyset, w)=\theta$, that is, $\Phi=w$.

\section{Fixed-Point Results of Expansive Maps}

Here, the case of expansive mappings in the setting of a $J$ -CMS over a BA is studied.

Theorem 32. Let $(\mathscr{M}, J)$ be a $\theta$-complete J-CMS over the BA $Y$ and $\chi: \mathscr{M} \longrightarrow \mathscr{M}$ be an onto mapping so that for all $\omega, \pi$ $\in \mathscr{M}$

$$
J\left(\chi \omega, \chi^{2} \omega, \chi \pi\right) \geqslant \alpha J(\omega, \chi \omega, \pi),
$$

where $\mathfrak{S} \mathfrak{R}\left(\alpha^{-1}\right)<1 / s$. Then, $\chi$ possesses a unique fixed point.

Proof. Let $\omega_{0} \in \mathscr{M}$. Since $\chi$ is onto, there is $\omega_{1} \in \mathscr{M}$ so that $\omega_{0}=\chi \omega_{1}$. By continuing this process, we get $\omega_{n}=\chi \omega_{n+1}$, for all $n \in N \cup\{0\}$. If $\omega_{n_{0}}=\omega_{n_{0+1}}$ for some $n_{0} \in N \cup\{0\}$, then $\omega_{n_{0}}$ is a fixed point of $\chi$. Assume that $\omega_{n} \neq \omega_{n+1}$ for all $n \in$ $N$. From (55), with $\omega=\omega_{n+1}$ and $\pi=\omega_{n}$, we have

$$
\begin{aligned}
J\left(\omega_{n}, \omega_{n-1}, \omega_{n-1}\right) & =J\left(\chi \omega_{n+1}, \chi^{2} \omega_{n+1}, \chi \omega_{n}\right) \\
& \geqslant \alpha J\left(\omega_{n+1}, \chi \omega_{n+1}, \omega_{n}\right) \\
& =\alpha J\left(\omega_{n+1}, \omega_{n}, \omega_{n}\right)
\end{aligned}
$$

which implies that

$$
J\left(\omega_{n+1}, \omega_{n}, \omega_{n}\right) \leqslant h J\left(\omega_{n}, \omega_{n-1}, \omega_{n-1}\right) \leqslant \cdots \leqslant h^{n} J\left(\omega_{0}, \omega_{1}, \omega_{1}\right),
$$

where $h=\alpha^{-1}$. Note that

$$
\mathfrak{S R}(h)=\mathfrak{S} \mathfrak{R}\left(\alpha^{-1}\right)<\frac{1}{s},
$$

so $(e-s h)^{-1}=\sum_{i=0}^{\infty}\left(s h^{i}\right)$ and $\left\|s h^{n}\right\| \longrightarrow 0(\infty)$. Following the lines of the proof of Theorem 29, we derive that $\left\{\omega_{n}\right\}$ is a $\theta$ -Cauchy sequence. Since $(\mathscr{M}, J)$ is $\theta$-complete, there is $\varpi \epsilon$ $\mathscr{M}$ so that $\omega_{n} \longrightarrow \Phi$, that is,

$$
\lim J\left(\omega_{n}, \Phi, \Phi\right)=\lim _{n, m \longrightarrow \infty} J\left(\omega_{n}, \omega_{m}, \omega_{m}\right)=J(\varrho, \emptyset, \emptyset)=\theta .
$$

Since $\chi$ is onto, there is $w \in \mathscr{M}$ so that $\emptyset=\chi w$. From (55) with $\omega=\omega_{n+1}$ and $\pi=w$, we have

$$
\begin{aligned}
J\left(\omega_{n}, \omega_{n-1}, \omega\right) & =J\left(\chi \omega_{n+1}, \chi^{2} \omega_{n+1}, \chi w\right) \geqslant \pm \alpha J\left(\omega_{n+1}, \chi \omega_{n+1}, w\right) \\
& =\alpha J\left(\omega_{n+1}, \omega_{n}, w\right) .
\end{aligned}
$$

So, $J\left(\omega_{n+1}, \omega_{n}, w\right) \leqslant h J\left(\omega_{n}, \omega_{n-1}, \emptyset\right)$.

Now, $\left\{J\left(\omega_{n}, \omega_{n-1}, \varpi\right)\right\}$ is a $c$-sequence; then, by using Lemma 25 and Lemma 26, it follows that $J(\emptyset, \emptyset, w)=\theta$. That is, $\Phi=w$. Then, $\Phi=\chi w=\chi \emptyset$. To prove the uniqueness, assume that $\emptyset \neq u$ so that $\chi u=u$ and $\chi \emptyset=\emptyset$. Now, by

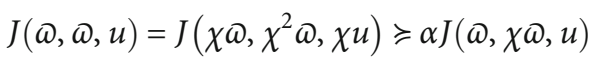

$$
\begin{aligned}
& \geqslant \alpha J(\varpi, \emptyset, u)>J(\varpi, \emptyset, u),
\end{aligned}
$$

which is a contradiction. Hence, $\emptyset=u$.

Theorem 33. Let $(\mathscr{M}, J)$ be a $\theta$-complete J-CMS over the BA $Y$ and $\chi: M \longrightarrow \mathscr{M}$ be so that for all $\omega, \pi \in \mathscr{M}$,

$$
J\left(\chi \omega, \chi \pi, \chi^{2} \pi\right) \geqslant \alpha J\left(\omega, \chi \omega, \chi^{2} \omega\right),
$$

where $\mathfrak{S} \mathfrak{R}\left(\alpha^{-1}\right)<1 / s$. Then, $\chi$ admits a unique fixed point.

Proof. Let $\omega_{0} \in \mathscr{M}$. Since $\chi$ is onto, there is $\omega_{1} \in \mathscr{M}$ so that $\omega_{0}=\chi \omega_{1}$. Let $\omega_{n}=\chi \omega_{n+1}$, for all $n \in N \cup\{0\}$. In case $\omega_{n_{0}}=$ $\omega_{n_{0+1}}$ for some $n_{0} \in N \cup\{0\}$, then $\omega_{n_{0}}$ is a fixed point of $\chi$. Suppose that $\omega_{n} \neq \omega_{n+1}$ for all $n \in N$. From (62) with $\omega=$ $\omega_{n+1}$ and $\pi=\omega_{n}$, we have

$$
J\left(\chi \omega_{n+1}, \chi \omega_{n}, \chi^{2} \omega_{n}\right) \geqslant \alpha J\left(\omega_{n+1}, \chi \omega_{n+1}, \chi^{2} \omega_{n+1}\right),
$$


which implies that

$$
J\left(\omega_{n}, \omega_{n-1}, \omega_{n-2}\right) \geqslant \alpha J\left(\omega_{n+1}, \omega_{n}, \omega_{n-1}\right),
$$

or

$$
J\left(\omega_{n+1}, \omega_{n}, \omega_{n-1}\right) \preccurlyeq \alpha^{-1} J\left(\omega_{n}, \omega_{n-1}, \omega_{n-2}\right) .
$$

Put $h=\alpha^{-1}$. It is evident that

$$
J\left(\omega_{n+1}, \omega_{n}, \omega_{n-1}\right) \leqslant h J\left(\omega_{n}, \omega_{n-1}, \omega_{n-2}\right),
$$

where $\mathfrak{S} \mathfrak{R}(h)<1 /$ s. By the mimic of the proof of Theorem 36 , we can show that $\omega_{n}$ is a $\theta$-Cauchy sequence. Since $(\mathscr{M}$, $J$ ) is a $\theta$-complete $J$-CMS over a BA, there is $\omega \in \mathscr{M}$ such that

$$
\lim J\left(\omega_{n}, \Phi, \Phi\right)=\lim _{n, m \longrightarrow \infty} J\left(\omega_{n}, \omega_{m}, \omega_{m}\right)=J(Ф, \Phi, \Phi)=\theta .
$$

Since $\chi$ is onto, there is $w \in \mathscr{M}$ so that $\Phi=\chi w$. From (62), we have for $\omega=w$ and $\pi=\omega_{n+1}$,

$$
J\left(\varrho, \omega_{n}, \omega_{n-1}\right)=J\left(\chi w, \chi \omega_{n+1}, \chi^{2} \omega_{n+1}\right) \geqslant \alpha J\left(w, \chi w, \chi^{2} w\right) .
$$

So, $J\left(w, \chi w, \chi^{2} w\right) \leqslant h J\left(\varpi, \omega_{n}, \omega_{n-1}\right)$,

where $h=\alpha^{-1}$. Since $\left\{J\left(\varrho, \omega_{n}, \omega_{n-1}\right)\right\}$ is a $c$-sequence, by using Lemma 25 and Lemma 26 , it follows that $J\left(\omega, \chi w, \chi^{2}\right.$ $w)=\theta$. That is, $w=\chi w=\chi^{2} w$.

\section{Examples}

The following examples demonstrate the results obtained in a $J$-CMS over Banach algebra $Y$.

Example 34. Take on $Y=C_{\mathbb{R}}^{1}[0,1]$ the norm $\|\omega\|=\|\omega\|_{\infty}+$ $\left\|\omega^{\prime}\right\|_{\infty}$ for $\omega \in Y$. Consider in $Y$ a just pointwise multiplication. Then, $Y$ is a real unit BA with unit $e=1$. Set the cone $\mathfrak{E}=\{\omega \in Y: \omega \geq 0\}$ in $Y$. Moreover, $\mathfrak{E}$ is not normal (see [12]). Let $\mathscr{M}=[0, \infty)$. Take $J: \mathscr{M} \times \mathscr{M} \times \mathscr{M} \longrightarrow Y$ as

$$
J(\omega, \pi, \omega)(t)=\left\{\begin{array}{ll}
\theta & , \text { if } \omega=\pi=\omega \\
\left(\max \{\omega, \pi, \omega\}^{2} e^{t}\right) & , \text { otherwise }
\end{array}= \begin{cases}\theta & , \text { if } \omega=\pi=\omega \\
\left(\max \left\{\omega^{2}, \pi^{2}, \omega^{2}\right\} e^{t}\right) & , \text { otherwise }\end{cases}\right.
$$

be a $J$-CMS over a BA on $\mathscr{M}$. Define $\chi: \mathscr{M} \longrightarrow \mathscr{M}$ by $\chi \omega$ $=(\omega / 5) \sin (\omega / 5)$. We have

$$
\begin{aligned}
J(\chi \omega, \chi \pi, \chi \pi)(t) & =J\left(\frac{\omega}{5} \sin \frac{\omega}{5}, \frac{\pi}{5} \sin \frac{\pi}{5}, \frac{\pi}{5} \sin \frac{\pi}{5}\right)(t) \\
& \leqslant \max \left\{\left(\frac{\omega}{5}\right)^{2},\left(\frac{\pi}{5}\right)^{2},\left(\frac{\pi}{5}\right)^{2}\right\} e^{t}=\frac{1}{25} \max \left\{\omega^{2}, \pi^{2}\right\} e^{t} .
\end{aligned}
$$

Therefore, $J(\chi \omega, \chi \pi, \chi \pi)(t) \preccurlyeq(1 / 25) J(\omega, \omega, \pi)(t)$. Then, the contractive condition (21) holds, and $\theta$ is the unique fixed point of $\chi$. That is, the conditions of Theorem 36 hold.

Example 35. Let $Y$ be a BA and $\mathfrak{C}$ be a cone (the same ones as those in Example 8), and let $\mathscr{M}=\mathbb{R}^{+}$. Consider $J: \mathscr{M} \times \mathscr{M}$ $\times \mathscr{M} \longrightarrow Y$ as in Example 8. Then, $(\mathscr{M}, J)$ is a $J$-CMS over the BA Y. Define $\chi: \mathscr{M} \longrightarrow \mathscr{M}$ by $\chi \omega=2 \omega$. Choose $\alpha=4$, then all the conditions of Theorem 6 hold and $\theta$ is the unique fixed point of $\chi$. Indeed,

$$
\begin{aligned}
J\left(\chi \omega, \chi^{2} \omega, \chi \pi\right)(t)= & \left(\left(\max \left\{\chi \omega, \chi^{2} \omega\right\}^{2}\right)+\left(\max \left\{\chi^{2} \omega, \chi \pi\right\}^{2}\right)\right. \\
& \left.+\left(\max \{\chi \pi, \chi \omega\}^{2}\right)\right) e^{t} \\
= & \left(\left(\max \{2 \omega, 4 \omega\}^{2}\right)+\left(\max \{4 \omega, 2 \pi\}^{2}\right)\right. \\
& \left.+\left(\max \{2 \pi, 2 \omega\}^{2}\right)\right) e^{t} \\
= & \left(16 \omega^{2}+4(\max \{2 \omega, \pi\})^{2}+4(\max \{\omega, \pi\})^{2}\right) e^{t} \\
= & 4\left(4 \omega^{2}+(\max \{2 \omega, \pi\})^{2}+(\max \{\omega, \pi\})^{2}\right) e^{t},
\end{aligned}
$$

$$
\begin{aligned}
J(\omega, \chi \omega, \pi)(t)= & \left(\left(\max \{\omega, \chi \omega\}^{2}\right)+\left(\max \{\chi \omega, \pi\}^{2}\right)\right. \\
& \left.+\left(\max \{\pi, \omega\}^{2}\right)\right) e^{t}, \\
= & \left(\left(\max \{\omega, 2 \omega\}^{2}\right)+\left(\max \{2 \omega, \pi\}^{2}\right)\right. \\
& \left.+\left(\max \{\pi, \omega\}^{2}\right)\right) e^{t}, \\
= & \left(4 \omega^{2}+(\max \{2 \omega, \pi\})^{2}+(\max \{\omega, \pi\})^{2}\right) e^{t},
\end{aligned}
$$

and so, $J\left(\chi \omega, \chi^{2} \omega, \chi \pi\right)(t) \geqslant 4 J(\omega, \chi \omega, \pi)(t)$.

\section{Application to the Existence of a Solution of Integral Equations}

Denote by $\mathscr{C}([0, T], \mathbb{R})$ the class of all continuous functions on $[0, T]$ (where $T>0$ ).

Let $Y=C[0, T]$ be considered with the norm $\|\omega\|=$ $\|\omega\|_{\infty}+\left\|\omega^{\prime}\right\|_{\infty}$. Take the usual multiplication, then $Y$ is a BA with the unit 1 . Set $\mathfrak{E}=\{\omega \in Y: \omega(t) \geq 0, t \in[a, b]\}$.

Let $J$ be the $J$-cone metric given as

$$
J(\rho, \varrho, \sigma)(t)=\sup _{t \in[0, T]}\left(|\rho(t)-\mathrm{Q}(t)|^{2}+|\mathrm{\varrho}(t)-\sigma(t)|^{2}+|\rho(t)-\mathrm{\varrho}(t)|^{2}\right) e^{t},
$$

for all $\rho, \varrho, \sigma \in \mathscr{C}([0, T], \mathbb{R})$. Note that $(\mathscr{C}([0, T], \mathbb{R}), J)$ is a complete $J$-CMS over BA $\mathscr{C}([0, T], \mathbb{R})$. 
Theorem 36. Let $K, f$, and a be continuous mappings so that:

(i) $\sup _{t \in[0, T]}|K(t, s)|=M<1 / \sqrt{T}$, where $K \in \mathscr{C}([0, T]$
$\quad \times[0, T], \mathbb{R})$

(ii) $a \in \mathscr{C}([0, T], \mathbb{R})$

(iii) $f \in \mathscr{C}([0, T] \times \mathbb{R}, \mathbb{R})$

(iv) $|f(s, \rho(s))-f(s, \rho(s))| \leq \zeta|\rho(s)-\mathrm{\varrho}(s)|$

for all $\rho, \varrho \in \mathscr{C}([0, T], \mathbb{R}), t \in[0, T]$, where $\zeta \in[0,1 / s)$. Then, the integral equation

$$
\rho(t)=\int_{0}^{T} K(t, s)(f(s, \rho(s))) d s+a(t), t \in[0, T]
$$

admits a unique solution in $\mathscr{C}([0, T], \mathbb{R})$.

Proof. Let $\mathscr{F}: \mathscr{C}([0, T], \mathbb{R}) \longrightarrow \mathscr{C}([0, T], \mathbb{R})$ be defined by

$$
\mathscr{F}(\rho)(t)=\int_{0}^{T} K(t, s) f(s, \rho(s)) d s+a(t)
$$

for all $t \in[0, T]$. We have

$$
\begin{aligned}
J(\mathscr{F}(\rho), \mathscr{F}(\mathrm{\varrho}), F(\mathrm{\varrho}))(t) & =\sup _{t \in[0, T]}\left(|\mathscr{F}(\rho(t))-\mathscr{F}(\mathrm{\varrho}(t))|^{2}+|\mathscr{F}(\mathrm{\varrho}(t))-\mathscr{F}(\mathrm{\varrho}(t))|^{2}+\mid \mathscr{F}(\rho(t))\right. \\
& \left.-\left.\mathscr{F}(\rho(t))\right|^{2}\right) e^{t}=2 \sup _{t \in[0, T]}\left(|\mathscr{F}(\rho(t))-\mathscr{F}(\rho(t))|^{2}\right) e^{t} \\
= & 2 \sup _{t \in[0, T]}\left(\int_{0}^{T}|K(t, s)|^{2}\left(|f(s, \rho(s))-f(s, \rho(s))|^{2}\right) d s\right) e^{t} \\
\leqslant & M^{2} T\left(2 \sup _{s \in[0, T]} \zeta|\rho(s)-\rho(s)|^{2}\right) e^{t}=\zeta J(\rho, \mathrm{\varrho}, \mathrm{\varrho}) .
\end{aligned}
$$

Then, all the conditions of Theorem 29 hold, and hence, there is a unique fixed point of $\mathscr{F}$. So, (73) has a unique solution.

\section{Data Availability}

No data were used to support this study.

\section{Conflicts of Interest}

The authors declare that they have no competing interests regarding the publication of this paper.

\section{Authors' Contributions}

All authors contributed equally and significantly in writing this article. All authors read and approved the final manuscript.

\section{References}

[1] N. Hussain, J. R. Roshan, V. Parvaneh, and A. Latif, "A unification of G-metric, partial metric and b-metric spaces," Abstract and Applied Analysis, vol. 2014, Article ID 180698, 14 pages, 2014.

[2] L. G. Huang and X. Zhang, "Cone metric spaces and fixed point theorems of contractive mappings," Journal of Mathematical Analysis and Applications, vol. 332, no. 2, pp. 14681476, 2007.

[3] H. Liu and S. Xu, "Cone metric spaces with Banach algebras and fixed point theorems of generalized Lipschitz mappings," Fixed point theory and applications, vol. 2013, no. 1, 2013.

[4] J. Fernandez, N. Malviya, D. Dolićanin-Dekić, and D. Pučić, "The $\mathrm{p}_{\mathrm{b}}$-cone metric spaces over Banach algebra with applications," Filomat, vol. 34, no. 3, 2020.

[5] H. Huang and S. Radenovic, "Common fixed point theorems of generalized Lipschitz mappings in cone metric spaces over Banach algebras," Applied Mathematics \& Information Sciences, vol. 9, no. 6, pp. 2983-2990, 2015.

[6] J. Fernandez, N. Malviya, and K. Saxena, "Cone b $b_{2}$-metric spaces over Banach algebra with applications," São Paulo Journal of Mathematical Sciences, vol. 11, pp. 221-239, 2017.

[7] M. Ahmad, A. Zada, and J. Alzabut, "Hyers-Ulam stability of a coupled system of fractional differential equations of HilferHadamard type," Demonstratio Mathematica, vol. 52, no. 1, pp. 283-295, 2019.

[8] A. Reinfelds and S. Christian, "A nonstandard Volterra integral equation on time scales," Demonstratio Mathematica, vol. 52, no. 1, pp. 503-510, 2019.

[9] M. Asadi, E. Karapinar, and P. Salimi, "A new approach to Gmetric and related fixed point theorems," Journal of Inequalities and Applications, vol. 2013, no. 1, Article ID 454, 2013.

[10] W. Rudin, Functional Analysis, McGraw-Hill, New York, 2nd edition, 1991.

[11] S. Xu and S. Radenović, "Fixed point theorems of generalized Lipschitz mappings on cone metric spaces over Banach algebras without assumption of normality," Fixed Point Theory and Applications, vol. 2014, 2014.

[12] S. Janković, Z. Kadelburg, and S. Radenović, "On cone metric spaces: a survey," Nonlinear Analysis: Theory, Methods \& Applications, vol. 4, no. 7, pp. 2591-2601, 2011.

[13] M. Ughade and R. D. Daheriya, "Fixed point and common fixed point results for contraction mappings $\mathrm{G}_{\mathrm{b}}$-cone metric spaces," Gazi University Journal of Science, vol. 28, 2015.

[14] Z. Kadelburg and S. Radenovic, "A note on various types of cones and fixed point results in cone metric spaces," Asian Journal of Mathematics and Applications, vol. 2013, article ama0104, pp. 1-7, 2013. 